\title{
Expanding the Applicability of Stirling's Method under Weaker Conditions and Restricted Convergence Regions
}

\author{
Ioannis K. Argyros ${ }^{1}$ and P. K. Parida ${ }^{2}$
}

\begin{abstract}
In this paper we have provided sufficient conditions to study semilocal and local convergence of the Stirling's method. The method is used to find fixed points of nonlinear operator equation. We assume Lipschtiz continuity type conditions on the first Fréchet derivative of the operator but no contractive conditions as in earlier works. This way expand the applicability of this method. Here we introduce a new type of majorizing sequences instead of usual majorizing sequences and recurrence relations. Finally the paper will be concluded with numerical examples and a favorable comparison with known results.
\end{abstract}

AMS Subject Classification (2000). 41A25; 47H10; 49M15; 65D10; 65Q05; 74G20; 74G30

Keywords. Stirling's method; Lipschtiz continuity condition; Majorizing sequences; Semilocal convergence; Local convergence, Computable radius of convergence

\footnotetext{
${ }^{1}$ Department of Mathematical Sciences, Cameron University, Lawton, USA, iargyros@cameron.edu

${ }^{2}$ Center for Applied Mathematics, Central University of Jharkhand, Ranchi, India, pkparida@cuj.ac.in
} 


\section{Introduction}

The present study is concerned with the semilocal and local approximations of an unique solution of nonlinear operator equations

$$
x=F(x),
$$

where $F$ is a nonlinear operator defined from $D$ subset of a Banach space $X$ with values in $X$. The given problem can be rechristened as the problem of approximating a fixed point $x^{*}$ of $F$. We can remember that the computation of solutions of nonlinear operator equations (1.1) is one of the most important and challenging problems in the field of numerical analysis, mathematical analysis, nonlinear functional analysis, mathematical physics and engineering etc. Also the given areas involve computation of fixed points of nonlinear equations. Numerous authors(see $[1,3,6,9-13,15,17-19]$ and references there in) have given different type of convergence conditions to approximate fixed points.

We can recall the problem of fixed points of nonlinear equations $F(x)=0$. The Banach theorems $[12,13]$ provide convergence of the successive approximation methods $x_{n+1}=F\left(x_{n}\right)$ to $x^{*}$ by assuming sufficient conditions on $F$. When $F$ is Fréchet differentiable at initial point $x_{0}$, Bartle [9] considered an iterative method of the form

$$
x_{n+1}=x_{n}-\left(I-F^{\prime}\left(y_{n}\right)\right)^{-1}\left(x_{n}-F\left(x_{n}\right)\right), \quad n=0,1,2, \ldots
$$

where $I$ is identity operator and $\left\{y_{n}\right\} \subset X$. The method reduces to usual Newton's method if we use $y_{n}=x_{n}$ and modified Newton's method if we choose $y_{n}=x_{0}$. One can see that if we choose $y_{n}=F\left(x_{n}\right)$ in Eq. (1.2), then Stirling's method [18] can be derived as given below

$$
x_{n+1}=x_{n}-\left(I-F^{\prime}\left(F\left(x_{n}\right)\right)\right)^{-1}\left(x_{n}-F\left(x_{n}\right)\right), n=0,1,2, \ldots
$$

The above method is a combination of Newton method and method of successive approximation methods. In the present paper we will study convergence of (1.3). Initially semilocal convergence of this method was studied by Rall [18] under Lipschitz continuity conditions and further the result was extended by Parhi and Gupta [14] by considering Hölder continuity conditions on $F^{\prime}$ under recurrence relations. By using majorizing sequence, Argyros [2] studied semilocal convergence of this method using Lipschitz-type conditions on $F^{\prime}$ and some additional informations on $F$. Parhi and Gupta $[15,16]$ studied semilocal convergence analysis under some restricted conditions on 
$F$. The most crucial hypothesis considered in these convergence analysis is the satisfaction of the contraction condition on $F^{\prime}$

$$
\left\|F^{\prime}(x)\right\|<1
$$

on $D$ and that is a major drawback in the usefulness of this method in finding fixed points. Let us consider the following example on $D=[-1,1]$

$$
F(x)=\frac{x^{2}}{2}
$$

It can be easily concluded that $\left\|F^{\prime}(x)\right\| \leq 1$, which restricts applicability of the work given in [1,2,14-16]. Recently Aryros et.al. [7] studied local and semilocal convergence of this method under Lipschitz-type conditions not necessarily contractive type.

In this paper we have studied semilocal and local convergence of Stirling's method by giving some sufficient conditions. The method is used to find fixed points of nonlinear operator equation. We have assumed Lipschitz continuity type conditions on the first Fréchet derivative of the operator not necessarily contractive type conditions on $F^{\prime}$. Here we will introduce a new type of majorizing sequences instead of usual majorizing sequences and recurrence relations. Finally the paper will be concluded with numerical examples and a favorable comparison with known results.

The paper is organized as follows. Section 1 is the introduction. In section 2, semilocal convergence of the method is given. The local convergence analysis of the method has been carried out in section 3 . In section 4 , numerical examples have been carried out and comparison has been done. Finally, conclusions are included in section 5 .

\section{Semilocal Convergence}

We first define a scalar sequence that shall be shown to be majorizing for Stirling's method (1.3). Let $L_{0}>0, L>0$ and $\eta \geq 0$. Define scalar sequence $\left\{t_{n}\right\}$ by

$$
t_{0}=0, t_{1}=\eta, t_{n+2}=t_{n+1}+\frac{L\left(t_{n+1}-t_{n}\right)^{2}}{2\left(1-L_{0} t_{n+1}\right)} .
$$

Next, we study the convergence of sequence $\left\{t_{n}\right\}$ using the preceding notation. 
Lemma 2.1. Suppose that

$$
h=L_{1} \eta<\frac{1}{2}
$$

where

$$
L_{1}=\frac{1}{8}\left(L+4 L_{0}+\sqrt{L^{2}+8 L_{0} L}\right) .
$$

Then, sequence $\left\{t_{n}\right\}$ generated for $t_{0}=0$ by (2.6) is increasing and converges to its unique least upper bound $t^{*}$. Moreover the following estimate holds

$$
d_{1} \leq t^{*} \leq d_{2}
$$

where,

$$
\begin{gathered}
d_{1}=\frac{1-\exp \left[-\frac{L_{0} \eta}{\left(1-L_{0} \eta\right)\left(1-\delta L_{0} \eta\right)}\right]}{L_{0}}, \\
d_{2}=\frac{1-\exp \left[\frac{2 L_{0} \eta}{2-L_{0} \eta}+\frac{2 \delta_{1}}{2-\delta_{1}}\right]}{L_{0}} \\
\delta=\frac{L}{2 L_{0}\left(1-L_{0} \eta\right)^{2}} \text { and } \delta_{1}=\frac{L}{2 L_{0}}\left(\frac{\delta}{1-\delta}\right)^{2} .
\end{gathered}
$$

Proof. We shall study the convergence of sequence $\left\{t_{n}\right\}$ by first simplifying it. Define sequence $\left\{\alpha_{n}\right\}$ by $\alpha_{n}=1-L_{0} t_{n}$. Then by (2.6) we can write

$$
\alpha_{0}=1, \alpha_{1}=1-L_{0} \eta, \alpha_{n+1}=\alpha_{n}-\frac{L\left(\alpha_{n}-\alpha_{n-1}\right)^{2}}{2 L_{0} \alpha_{n}} .
$$

Moreover, define sequence $\left\{\beta_{n}\right\}$ by $\beta_{n}=1-\frac{\alpha_{n}}{\alpha_{n-1}}$. Then, we can write

$$
\beta_{1}=L_{0} \eta, \beta_{n+1}=\frac{L}{2 L_{0}}\left(\frac{\beta_{n}}{1-\beta_{n}}\right)^{2} \text {. }
$$

We have by (2.6) that $\delta \alpha_{1}<1$ and $0<\alpha_{2}<\alpha_{1}$. Suppose that $0<\alpha_{k}<\alpha_{k-1}$ and $\delta \alpha_{k}<1$. Then, we get in turn that

$$
\alpha_{k+1}=\frac{L}{2 L_{0}}\left(\frac{\alpha_{k}}{1-\alpha_{k}}\right)^{2}<\delta \alpha_{k}^{2}<\alpha_{k}
$$

and

$$
\delta \alpha_{k+1}<\delta \alpha_{k}<1
$$


Hence, $\left\{\beta_{n}\right\}$ is a decreasing sequence, so $\alpha_{n}=\left(1-\beta_{n}\right) \alpha_{n-1}$ and $t_{n}=\frac{1-\alpha_{n}}{L_{0}}$ are also decreasing sequences. In particular,

$$
\begin{aligned}
\alpha_{n} & =\left(1-\beta_{n}\right) \alpha_{n-1}=\cdots=\left(1-\beta_{n}\right) \ldots\left(1-\beta_{1}\right) \alpha_{0} \\
& =\left(1-\beta_{n}\right) \ldots\left(1-\beta_{1}\right)
\end{aligned}
$$

From $0<\beta_{1}=L_{0} \eta<1$, we get $0<\alpha_{n}<1$. So $t_{n}=\frac{1-\alpha_{n}}{\gamma}<\frac{1}{\gamma}$. That is sequence $\left\{t_{n}\right\}$ is increasing, bounded by $\frac{1}{L_{0}}$ and as such it converges to its unique least upper bound denoted by $t^{*}$. Next, we show (2.7). We can write

$$
\alpha^{*}=\lim _{n \rightarrow \infty} \alpha_{n}=\prod_{n=1}^{\infty}\left(1-\beta_{n}\right),
$$

or

$$
\log \frac{1}{\alpha^{*}}=\sum_{n=1}^{\infty} \log \frac{1}{1-\beta_{n}}
$$

Using the estimate

$$
2 \frac{t-1}{t+1} \leq \log t \leq \frac{t^{2}-1}{2 t} \text { for } t>1
$$

we get first an upper bound for $\log \frac{1}{\alpha^{*}}$ by $(2.8)$ and (2.9) and the inequality $2^{n} \geq n+1$ for $n=0,1,2, \ldots$.

$$
\begin{aligned}
\log \frac{1}{\alpha^{*}} & \leq \sum_{n=1}^{\infty} \frac{\beta_{n}\left(2-\beta_{n}\right)}{2\left(1-\beta_{n}\right)} \leq \frac{1}{1-\beta_{1}} \sum_{n=0}^{\infty} \beta_{n+1} \\
& \leq \frac{1}{\delta\left(1-\beta_{1}\right)} \sum_{n=1}^{\infty}\left(\delta \theta_{1}\right)^{2^{n}} \leq \frac{1}{\delta\left(1-\beta_{1}\right)} \sum_{n=1}^{\infty}\left(\delta \beta_{1}\right)^{n} \\
& =\frac{\beta_{1}}{\left(1-\beta_{1}\right)\left(1-\delta \beta_{1}\right)}
\end{aligned}
$$

which together with $t^{*}=\frac{1-\alpha^{*}}{L_{0}}$ imply $t^{*} \leq d_{2}$. The lower bound in (2.7) is obtained simplifying the estimate:

$$
\log \frac{1}{\alpha^{*}} \geq 2 \sum_{n=1}^{\infty} \frac{\alpha_{n}}{2-\alpha_{n}}>\frac{2 \alpha_{1}}{2-\alpha_{1}}+\frac{2 \alpha_{2}}{2-\alpha_{2}} .
$$


Lemma 2.2. Suppose that

$$
h=\frac{1}{2}
$$

Then, sequence $\left\{t_{n}\right\}$ is increasingly converging to $\frac{1}{L_{0}}$.

Proof. We have $\alpha_{n}=\left(1-L_{0} \eta\right)^{n}, \beta_{n}=L_{0} \eta$ and $t_{n}=\frac{1-\left(1-L_{0} \eta\right)^{n}}{L_{0}}$. Then, by (2.10), we get $0<L_{0} \eta<1$.

The semilocal convergence analysis is based on the conditions $(\mathrm{C})$ :

$\left(C_{1}\right) F: D \subset X \rightarrow X$ is a Fréchet differentiable operator and there exist $x_{0} \in D, c>0, \eta \geq 0$ such that $\Gamma_{0}=\Gamma\left(x_{0}\right)=\left(I-F^{\prime}\left(F\left(x_{0}\right)\right)\right)^{-1} \in$ $L(X, X)$ with $\left\|I-F^{\prime}\left(F\left(x_{0}\right)\right)\right\| \leq c$ and $\left\|\Gamma_{0}\left(x_{0}-F\left(x_{0}\right)\right)\right\| \leq \eta$.

$\left(C_{2}\right)$ There exist $a_{0} \in[0,1), b_{0}>0$ such that for each $x \in D, \| F(x)-$ $F\left(x_{0}\right)\left\|\leq a_{0}\right\| x-x_{0} \|$ and $\left\|\Gamma_{0}\left(F^{\prime}(F(x))-F^{\prime}\left(F\left(x_{0}\right)\right)\right)\right\| \leq b_{0} \| F(x)-$ $F\left(x_{0}\right) \|$.

$\left(C_{3}\right)$ Let $D_{0}=D \bigcap U\left(x_{0}, r_{0}\right), r_{0}=\frac{1}{a_{0} b_{0}}$. There exist $b>0, b_{1}>0$ such that for each $x, y \in D_{0},\left\|\Gamma_{0}\left(F^{\prime}(x)-F^{\prime}(y)\right)\right\| \leq b\|x-y\|$ and $\| F^{\prime}(F(x))-$ $F^{\prime}\left(F\left(x_{0}\right)\right)\left\|\leq b_{1}\right\| F(x)-F\left(x_{0}\right) \|$.

$\left(C_{4}\right)$ Hypotheses of Lemma 2.1 and Lemma 2.2 hold with $L=2 b\left(c+\frac{b_{1}}{b_{0}}+\frac{1}{2}\right)$ and $L_{0}=\eta_{0} b_{0}$.

$\left(C_{5}\right) \frac{\left\|F\left(x_{0}\right)-x_{0}\right\|}{1-a_{0}} \leq t^{*}$.

$\left(C_{6}\right) \bar{U}\left(x_{0}, t^{*}\right) \subseteq D$.

From now on we suppose that the conditions $(C)$ hold. The main semilocal convergence result for Stirling's method (1.3) is given next.

Theorem 2.1. Suppose that the conditions (C) hold. Then, sequence $\left\{x_{n}\right\}$ generated by Stirling's method (1.3) is well defined, remains in $U\left(x_{0}, t^{*}\right)$ for each $n=0,1,2, \ldots$ and converges to $x^{*} \in \bar{U}\left(x_{0}, t^{*}\right)$ which satisfies $x^{*}=F\left(x^{*}\right)$ with $Q$-order of convergence 2. Moreover, the following estimates hold

$$
\left\|x_{n}-x^{*}\right\| \leq t^{*}-t_{n}
$$

Furthermore, $x^{*}$ is the only fixed point of $F$ in $U\left(x_{0}, t^{* *}\right)$, where $t^{* *}=\frac{2}{b}-$ $\left(2 \alpha_{0}+1\right) t^{*}$. 
Proof. Let $x \in \bar{U}\left(x_{0}, t^{*}\right)$. We get by $\left(C_{2}\right)$ and $\left(C_{5}\right)$ that

$$
\begin{aligned}
\left\|F(x)-x_{0}\right\| & \leq\left\|F(x)-F\left(x_{0}\right)\right\|+\left\|F\left(x_{0}\right)-x_{0}\right\| \\
& \leq \alpha_{0}\left\|x-x_{0}\right\|+\left\|F\left(x_{0}\right)-x_{0}\right\| \\
& \leq \alpha_{0} t^{*}+\left\|F\left(x_{0}\right)-x_{0}\right\| \leq t^{*}
\end{aligned}
$$

so $F(x) \in \bar{U}\left(x_{0}, t^{*}\right)$. Using $\left(C_{2}\right)$ and the Lemmas, we have in turn that

$$
\begin{aligned}
\left\|\Gamma_{0}\left(\Gamma(x)-\Gamma_{0}\right)\right\| & =\left\|\Gamma_{0}\left(F^{\prime}(F(x))-F^{\prime}\left(F\left(x_{0}\right)\right)\right)\right\| \\
& \leq b_{0} a_{0}\left\|x-x_{0}\right\|=L_{0}\left\|x-x_{0}\right\| \leq L_{0} t^{*}<1 .
\end{aligned}
$$

It follows from (2.11) and the Banach Lemma on invertible operators $[4,8]$ that $\Gamma(x)^{-1} \in L(X, X)$ and

$$
\left\|\Gamma(x)^{-1}\left(I-F^{\prime}\left(F\left(x_{0}\right)\right)\right)\right\| \leq \frac{1}{1-L_{0}\left\|x-x_{0}\right\|}
$$

We can write by Stirling's method (1.3):

$$
\begin{aligned}
x_{k+1}-F\left(x_{k+1}\right)= & F\left(x_{k+1}\right)-F\left(x_{k}\right)-F\left(x_{k+1}\right)+F\left(x_{k}\right) \\
= & F^{\prime}\left(y_{k}\right)\left(x_{k+1}-x_{k}\right)-\left(F\left(x_{k+1}\right)-F\left(x_{k}\right)\right) \\
= & \int_{0}^{1}\left[F^{\prime}\left(y_{k}\right)-F^{\prime}\left(x_{k}+\theta\left(x_{k+1}-x_{k}\right)\right)\right] \\
& \times\left(x_{k+1}-x_{k}\right) d \theta .
\end{aligned}
$$

Then, in view of $\left(C_{2}\right),\left(C_{3}\right)$ and $(2.13)$, we obtain in turn that

$$
\begin{aligned}
\left\|\Gamma_{0}\left(x_{k+1}-F\left(x_{k+1}\right)\right)\right\| \leq & b \int_{0}^{1}\left\|y_{k}-x_{k}-\theta\left(x_{k+1}-x_{k}\right)\right\|\left\|x_{k+1}-x_{k}\right\| d \theta \\
\leq & b\left[\left\|y_{k}-x_{k}\right\|+\frac{1}{2}\left\|x_{k+1}-x_{k}\right\|\right]\left\|x_{k+1}-x_{k}\right\| \\
\leq & b\left[\left(\left\|I-F^{\prime}\left(F\left(x_{0}\right)\right)\right\|+\| F^{\prime}\left(y_{k}\right)\right.\right. \\
& \left.-F^{\prime}\left(F\left(x_{0}\right)\right) \|\right)\left\|x_{k+1}-x_{k}\right\| \\
& \left.+\frac{1}{2}\left\|x_{k+1}-x_{k}\right\|\right]\left\|x_{k+1}-x_{k}\right\| \\
\leq & b\left(c+\frac{b_{1} a_{0}}{a_{0} b_{0}}+\frac{1}{2}\right)\left\|x_{k+1}-x_{k}\right\|^{2} \\
= & \frac{L}{2}\left\|x_{k+1}-x_{k}\right\|^{2} .
\end{aligned}
$$


Now, we can connect the preceding estimates on sequence $\left\{x_{k}\right\}$ with $\left\{t_{k}\right\}$. Indeed, we get by $\left(C_{1}\right)$ and (2.6) that

$$
\left\|x_{1}-x_{0}\right\|=\left\|\Gamma_{0}\left(x_{0}-F\left(x_{0}\right)\right)\right\| \leq \eta=t_{1}=t_{1}-t_{0} .
$$

By induction, from (1.3), (2.6), (2.12) and (2.14), we have in turn that

$$
\begin{aligned}
\left\|x_{k+1}-x_{k}\right\| & =\left\|\Gamma_{k}\left(x_{k}-F\left(x_{k}\right)\right)\right\| \\
& \leq\left\|\Gamma_{k}\left(I-F^{\prime}\left(F\left(x_{0}\right)\right)\right)\right\|\left\|\Gamma_{0}\left(x_{k}-F\left(x_{k}\right)\right)\right\| \\
& \leq \frac{L}{2} \frac{\left(t_{k}-t_{k-1}\right)^{2}}{1-L_{0} t_{k}}=t_{k+1}-t_{k} .
\end{aligned}
$$

Hence, $\left\{t_{k}\right\}$ defined by (2.6) is a majorizing sequence for $\left\{x_{k}\right\}$. By Lemma 2.1 and Lemma 2.2, sequence $\left\{t_{k}\right\}$ is complete as convergent to $t^{*}$. It then follows by (2.15) that sequence $\left\{x_{k}\right\}$ is also complete in a Banach space $X$ and as such it converges to some $x^{*} \in \bar{U}\left(x_{0}, t^{*}\right)$ (since $\bar{U}\left(x_{0}, t^{*}\right)$ is a closed set). By letting $k \rightarrow \infty$ in the estimate (see (2.14))

$$
\left\|\Gamma_{0}\left(x_{k+1}-F\left(x_{k+1}\right)\right)\right\| \leq \frac{L}{2}\left\|x_{k+1}-x_{k}\right\|^{2} \leq \frac{L}{2}\left(t_{k+1}-t_{k}\right)^{2},
$$

we deduce that $x^{*}=F\left(x^{*}\right)$. Estimate $\left\|x_{n}-x^{*}\right\| \leq t^{*}-t_{n}$ follows from (2.15) by using standard majorization techniques $[6,13]$. Moreover, by $(2.15)$ for $\lambda=\frac{L}{2\left(1-L_{0} t^{*}\right)}$ we get that

$$
\left\|x_{k+1}-x_{k}\right\| \leq \frac{L}{2\left(1-L_{0} t_{k}\right)}\left\|x_{k}-x_{k-1}\right\|^{2} \leq \lambda\left\|x_{k}-x_{k-1}\right\|^{2},
$$

which implies that the $Q$-order convergence of Stirling's method (1.3) is two. Furthermore, to show the uniqueness part, let $y^{*} \in U\left(x_{0}, t^{* *}\right)$ with $F\left(y^{*}\right)=y^{*}$. Define the operator $Q$ by $Q=\int_{0}^{1} \Gamma_{0} F^{\prime}\left(x^{*}+\theta\left(y^{*}-x^{*}\right)\right) d \theta$. In view of $\left(C_{2}\right)$ and $\left(C_{3}\right)$ we obtain in turn that

$$
\begin{aligned}
\left\|I-\left(\Gamma_{0}-Q\right)\right\| & =\left\|\int_{0}^{1} \Gamma_{0}\left[F^{\prime}\left(x^{*}+\theta\left(y^{*}-x^{*}\right)\right)-F^{\prime}\left(F\left(x_{0}\right)\right)\right] d \theta\right\| \\
& \leq b \int_{0}^{1}\left\|x^{*}+\theta\left(y^{*}-x^{*}\right)-F\left(x_{0}\right)\right\| d \theta \\
& \leq b\left[\left\|F\left(x^{*}\right)-F\left(x_{0}\right)\right\|+\frac{1}{2}\left\|x^{*}-x_{0}\right\|+\frac{1}{2}\left\|y^{*}-x_{0}\right\|\right] \\
& \leq b\left[\left(a_{0}+\frac{1}{2}\right) t^{*}+\frac{1}{2} t^{* *}\right]<1 .
\end{aligned}
$$


Then, by (2.17), $\left(\Gamma_{0}-Q\right)^{-1} \in L(X, X)$. Finally, from the identity

$$
0=\Gamma_{0}\left(y^{*}-F\left(y^{*}\right)-x^{*}+F\left(x^{*}\right)\right)=\left(\Gamma_{0}-Q\right)\left(y^{*}-x^{*}\right),
$$

we conclude that $x^{*}=y^{*}$.

Remark 2.1. (a) The usual conditions on Stirling's method corresponding to $\left(C_{2}\right)$ (first condition) is given by [14,18]:

$\left(C_{2}\right)^{\prime}\left\|F^{\prime}(x)\right\| \leq a$, for each $x \in D$ and $a \in[0,1)$. That is operator $F$ must be a contraction on D. Moreover, in the present study no such assumption is made. Hence, the applicability of Stirling's method (1.3) is expanded. Notice also that we can have $a_{0} \leq a, b_{0} \leq b$, where $b$ can be chosen to be $b=c b_{1}$.

(b) Estimate (2.7) is similar to the sufficient convergence Kantorovich-type condition for the semilocal convergence of Newton's method given by us in [5]. However, the constants $L_{0}$ and $L$ are the center-Lipschitz and Lipschitz constants for operator $F$. The convergence of Stirling's method was shown in [18] under $\left(C_{2}\right)^{\prime}, D_{0}=D$ and $a \in\left(0, \frac{1}{3}\right)$. Here, no such hypothesis is made.

(c) The results can be improved even further, if set $D_{0}$ is replaced by $D_{1}=$ $D \bigcap U\left(x_{1}, r_{0}-\left\|x_{0}-F\left(x_{0}\right)\right\|\right)$, since $D_{1} \subseteq D$ and the iterates remain in $D_{1}$. The corresponding constants to $b$ and $b_{1}$ will be at least as small.

(d) In view of the proof of Theorem 2.1, scalar sequence $\left\{s_{n}\right\}$ defined by

$$
s_{0}=0, s_{1}=\eta, s_{n+1}=s_{n}+\frac{k_{n}\left(s_{n}-s_{n-1}\right)^{2}}{1-L_{0} s_{n}}
$$

is also a majorizing sequence for Stirling's method (1.3), where $k_{n}=2 b(c+$ $\left.b_{1} a_{0} s_{n}+\frac{1}{2}\right)<L, s_{n} \leq t_{n}, s_{n+1}-s_{n} \leq t_{n+1}-t_{n}$ and $s^{*}=\lim _{n \rightarrow \infty} s_{n} \leq t^{*}$.

\section{Local Convergence}

The local convergence analysis of Stirling's method (1.3) is based on the conditions $(\mathrm{H})$ :

$\left(H_{1}\right) F: D \subset X \rightarrow Y$ is a Fréchet differentiable operator and there exists $x^{*} \in D$ such that $F\left(x^{*}\right)=x^{*}$ and $\Gamma_{*}=\left(I-F^{\prime}\left(x^{*}\right)\right)^{-1} \in L(X, X)$

$\left(H_{2}\right)$ There exist $\mu>0, \xi_{0}>0$ such that for each $x \in D$

$$
\left\|F(x)-F\left(x^{*}\right)\right\| \leq \mu\left\|x-x^{*}\right\|
$$

and

$$
\| \Gamma_{*}\left(F^{\prime}(F(x))-F^{\prime}\left(F\left(x^{*}\right)\right)\left\|\leq \xi_{0}\right\| F(x)-F\left(x^{*}\right) \|\right.
$$


$\left(H_{3}\right)$ Let $D_{0}^{*}=D \bigcap U\left(x^{*}, R_{0}\right), R_{0}=\frac{1}{\xi_{0} \mu}$. There exists $\xi>0$ such that for each $x, y \in D_{0}^{*}$

$$
\left\|\Gamma_{*}\left(F^{\prime}(x)-F^{\prime}(y)\right)\right\| \leq \xi\|x-y\|
$$

$\left(H_{4}\right) \bar{U}\left(x^{*}, \bar{R}\right) \subseteq D$, where $R=\frac{1}{\left(\mu+\frac{1}{2}\right) \xi+\mu \xi_{0}}$ and $\bar{R}=\max \{\mu R, R\}$.

Then, sequence $\left\{x_{n}\right\}$ generated for $x_{0} \in U\left(x^{*}, R\right)-\left\{x^{*}\right\}$ by Stirling's method (1.3) is well defined, remains in $U\left(x^{*}, R\right)$ for each $n=0,1,2, \ldots$ and converges to $x^{*} \in \bar{U}\left(x^{*}, R\right)$. Moreover, the following estimates hold

$$
\left\|x_{n+1}-x^{*}\right\| \leq \frac{\xi\left(\mu+\frac{1}{2}\right)\left\|x_{n}-x^{*}\right\|^{2}}{1-\mu \xi_{0}\left\|x_{n}-x^{*}\right\|} .
$$

Furthermore, if $R_{1}=\frac{2}{\xi}$, then $x^{*}$ is the only fixed point of $F$ on $U\left(x^{*}, R_{1}\right)$

Proof. We shall show using mathematical induction that sequence $\left\{x_{n}\right\}$ is well defined, remains in $U\left(x^{*}, R\right)$ and converges to $x^{*}$ so that (3.18) is satisfied. We have by $\left(H_{1}\right)$ and $\left(H_{2}\right)$ for $x_{0} \in U\left(x^{*}, R\right)$ that

$$
\left\|F\left(x_{0}\right)-x^{*}\right\|=\left\|F\left(x_{0}\right)-F\left(x^{*}\right)\right\| \leq \mu\left\|x_{0}-x^{*}\right\| \leq \bar{R}
$$

so $F\left(x_{0}\right) \in U\left(x^{*}, \bar{R}\right)$. Then, by $\left(H_{2}\right)$

$$
\begin{aligned}
\left\|\Gamma_{*}\left(I-F^{\prime}\left(F\left(x_{0}\right)\right)-\Gamma_{*}\right)\right\| & =\| \Gamma_{*}\left(F^{\prime}\left(F\left(x_{0}\right)\right)-F^{\prime}\left(F\left(x^{*}\right)\right) \|\right. \\
& \leq \xi_{0}\left\|F\left(x_{0}\right)-F\left(x^{*}\right)\right\| \\
& \leq \xi_{0} \mu\left\|x_{0}-x^{*}\right\| \leq \xi_{0} \mu R<1 .
\end{aligned}
$$

Hence, $\Gamma\left(x_{0}\right) \in L(X, X)$ and

$$
\left\|\Gamma\left(x_{0}\right)\left(I-F\left(F\left(x^{*}\right)\right)\right)\right\| \leq \frac{1}{1-\xi_{0} \mu\left\|x_{0}-x^{*}\right\|}
$$

In particular, $x_{1}$ is well defined by Stirling's method for $n=0$. We can write by $\left(H_{1}\right)$ that

$$
\begin{aligned}
x_{1}-x^{*}= & x_{0}-x^{*}-\left(I-F^{\prime}\left(F\left(x_{0}\right)\right)\right)^{-1}\left(x_{0}-F\left(x_{0}\right)\right) \\
= & \left(I-F^{\prime}\left(F\left(x_{0}\right)\right)\right)^{-1}\left[F\left(x_{0}\right)-F\left(x^{*}\right)-F^{\prime}\left(F\left(x_{0}\right)\right)\left(x_{0}-x^{*}\right)\right] \\
= & \left(I-F^{\prime}\left(F\left(x_{0}\right)\right)\right)^{-1}\left[\int _ { 0 } ^ { 1 } \left(F^{\prime}\left(x^{*}+\theta\left(x_{0}-x^{*}\right)\right)\right.\right. \\
& \left.\left.-F^{\prime}\left(F\left(x_{0}\right)\right)\right)\left(x_{0}-x^{*}\right) d \theta\right] .
\end{aligned}
$$


We get in turn by $\left(H_{2}\right)$ and $\left(H_{3}\right)$

$$
\begin{aligned}
& \left\|\Gamma_{*} \int_{0}^{1}\left(F^{\prime}\left(x^{*}+\theta\left(x_{0}-x^{*}\right)\right)-F^{\prime}\left(F\left(x_{0}\right)\right)\right)\left(x_{0}-x^{*}\right) d \theta\right\| \\
\leq & \xi \int_{0}^{1}\left\|x^{*}+\theta\left(x_{0}-x^{*}\right)-F\left(x_{0}\right)\right\|\left\|x_{0}-x^{*}\right\| d \theta \\
\leq & \xi \int_{0}^{1}\left\|F\left(x^{*}\right)-F\left(x_{0}\right)+\theta\left(x_{0}-x^{*}\right)\right\|\left\|x_{0}-x^{*}\right\| d \theta \\
\leq & \xi\left(\mu+\frac{1}{2}\right)\left\|x_{0}-x^{*}\right\|^{2} .
\end{aligned}
$$

Then by (3.20)-(3.22), we also get that

$$
\begin{aligned}
\left\|x_{1}-x^{*}\right\| \leq & \left\|\left(I-F^{\prime}\left(F\left(x_{0}\right)\right)\right)^{-1} \Gamma_{*}\right\| \times \\
& \left\|\Gamma_{*} \int_{0}^{1}\left(F^{\prime}\left(x^{*}+\theta\left(x_{0}-x^{*}\right)\right)-F^{\prime}\left(F\left(x_{0}\right)\right)\right)\left(x_{0}-x^{*}\right) d \theta\right\| \\
\leq & \frac{\xi\left(\mu+\frac{1}{2}\right)\left\|x_{0}-x^{*}\right\|^{2}}{1-\mu \xi_{0}\left\|x_{0}-x^{*}\right\|} \leq\left\|x_{0}-x^{*}\right\|<R,
\end{aligned}
$$

so (3.18) holds for $n=0$ and $x_{1} \in U\left(x^{*}, R\right)$. By simply replacing $x_{0}$ by $x_{k}$ in the preceding estimates we arrive at (3.18). In view of the estimate $\left\|x_{k+1}-x^{*}\right\| \leq c\left\|x_{k}-x^{*}\right\|<R$, where $c=\frac{\xi\left(\mu+\frac{1}{2}\right)\left\|x_{0}-x^{*}\right\|}{1-\mu \xi_{0}\left\|x_{0}-x^{*}\right\|} \in[0,1)$, we conclude that $\lim _{k \rightarrow \infty} x_{k}=x^{*}$ and $x_{k+1} \in U\left(x^{*}, R\right)$. To show the uniqueness part let $x_{0}=x^{*}$ in $(2.17)$.

Remark 3.1. The local results in the literature use $\left(C_{2}\right)^{\prime}$ and $D_{0}^{*}=D$. But $\left(H_{2}\right)$ is weaker than $\left(C_{2}\right)^{\prime}$. Hence, we extend the applicability of Stirling's method (1.3) in the local case too.

\section{$4 \quad$ Numerical Examples}

In this section we present two numerical examples to illustrate our results.

Example 4.1. Again coming back to the motivational example given in Eq. (1.5), let initial point be $x_{0}=0.1$. Then we can have $c=0.995000, \eta=$ $0.095477, a_{0}=0.505, b_{0}=1.005025, r_{0}=1.970297, b=1.005025, b_{1}=1$. Using this we can have $L_{1} \eta=0.128465<\frac{1}{2}$. Therefore, hypothesis $(C)$ satisfies and our analysis can be applied and hence the radius of convergence $t^{*}$ is given by $t^{*}=0.119847$. 
But if we recall the results given by Argyros, Maruster and George [7] on Stirling's method, we can can see that the radius of convergence given by them is $R=0.02$. Therefore our analysis greatly improves the previous result.

Example 4.2. Let $B_{1}=B_{2}=\mathbb{R}^{3}, D=\bar{U}(0,1), x^{*}=(0,0,0)^{T}$. For $x=$ $\left(x_{1}, x_{2}, x_{3}\right)^{T}$, define function $G$ on $D$

$$
G(x)=\left(e^{x_{1}}-1, \frac{e-1}{2} x_{2}^{2}+x_{2}, x_{3}\right)^{T} .
$$

Then consider the problem of finding fixed points of $F(u)=G(u)+u$. Here we will use max-norm for our calculation.

Note that, the Fréchet-derivative of $G$ is given by

$$
G^{\prime}(u)=\left[\begin{array}{ccc}
e^{u_{1}} & 0 & 0 \\
0 & (e-1) u_{2}+1 & 0 \\
0 & 0 & 1
\end{array}\right]
$$

and $F^{\prime}(u)=G^{\prime}(u)+I$. Then, we can choose $\mu=e+1, \xi_{0}=e-1$ and $\xi=e^{\frac{1}{\xi_{0} \mu}}$. Therefore $R=0.08832324882$ and $\bar{R}=0.3284107311$. We can check that condition $\left(H_{4}\right)$ is satisfied. Hence the radius of convergence is $R=0.08832324882$

\section{Conclusions}

We have expanded the applicability of Stirling's method (1.3) by considering more precise majorizing sequences than in earlier works $[2,7]$ and without using the restrictive contraction hypothesis $[1,2,7,14-16]$. We provided numerical examples which shows applicability of our work whereas earlier works cannot apply to solve equations.

\section{References}

[1] I. K. Argyros, Stirling's method and fixed points of nonlinear operator equations in Banach spaces, Bull. Inst. Math. Acad. Sin. (N.S.) 23, (1995), 13-20

[2] I. K. Argyros, On The Convergence and Application of Stirling's Method, Applicationes Mathematicae, 30, (2003), 109-119

[3] I. K. Argyros, A new iterative method of asymptotic order $1+\sqrt{2}$ for the computation of fixed points, Int. J. Comput. Math. 82, (2005), 1413-1428 
[4] I. K. Argyros, Computational Theory of Iterative Methods. Series: Studies in Computational Mathematics, 15, (2007)

[5] I. K. Argyros and S. Hilout, Weaker conditions for the convergence of Newton's method, Journal of Complexity, 28, (2012), 364-387

[6] I. K. Argyros and A. A. Magreñán, Iterative Methods and Their Dynamics with Applications: A Contemporary Study, CRC Press, New York, 2017

[7] S. Maruster and S. George I. K. Argyros, On the Convergence of Stirling's Method for Fixed Points Under Not Necessarily Contractive Hypotheses, International Journal of Applied and Computational Mathematics, 3, (2017), 1071-1081

[8] I. K. Argyros and F. Szidarovszky, The Theory and Applications of Iteration Methods, CRC Press, Boca Raton, Florida, USA, 1993

[9] R. G. Bartle, Newton's method in Banach spaces, Proc. Amer. Math. Soc. 6, (1955), $827-831$

[10] S. K. Parhi and S. Singh D. K. Gupta, Semilocal convergence of Stirling's method for fixed points in Banach spaces, International Journal of Mathematics in Operational Research, 9, (2016), 243-257

[11] M. A. Hernández, The Newton Method for Operators with Hölder Continuous First Derivative, Journal of Optimization Theory and Applications, 109, (2001), 631-648

[12] L. V. Kantorovich and G. P. Akilov, Functional Analysis, Pergamon Press, Oxford, 1982

[13] J. M. Ortega and W. C. Rheinboldt, Iterative Solution of Nonlinear Equations in Several Variables, SIAM, Philadelphia, 2000

[14] S. K. Parhi and D. K. Gupta, Semilocal convergence of Stirling's method under Hölder continuous first derivative in Banach spaces, International Journal of Computer Mathematics, 87, (2010), 2752-2759

[15] S. K. Parhi and D. K. Gupta, Relaxing convergence conditions for Stirling's method, Mathematical methods in the Applied Sciences, 33, (2010), 224-232

[16] S. K. Parhi and D. K. Gupta, Convergence of Stirling's method under weak differentiability condition, Mathematical methods in the Applied Sciences, 34, (2011), $168-175$

[17] L. B. Rall, Computational Solution of Nonlinear Operator Equations, E. Robert Krieger, New York, 1969

[18] L. B. Rall, Convergence of Stirling's method in Banach spaces, Aequationes Math. 12, (1975), 12-20

[19] W. Werner, Newton-like methods for the computation of fixed points, Comput. Math. Appl. 10, (1984), 77-86

Received: 10.11.2017

Accepted: 10.01 .2018

Revised: 5.01.2018 